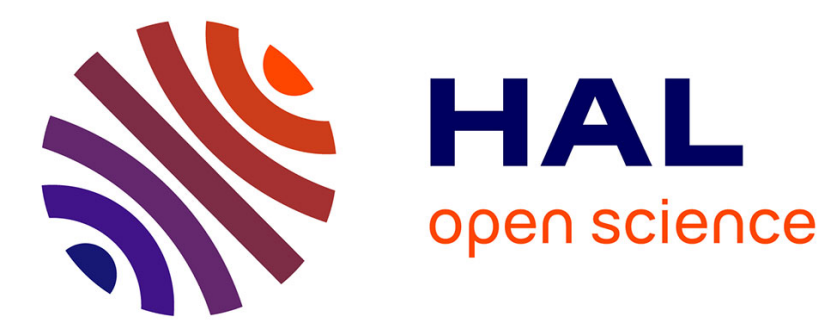

\title{
Assessment of CNN-based Methods for Poverty Estimation from Satellite Images
}

Robin Jarry, Marc Chaumont, Laure Berti-Équille, Gérard Subsol

\section{To cite this version:}

Robin Jarry, Marc Chaumont, Laure Berti-Équille, Gérard Subsol. Assessment of CNN-based Methods for Poverty Estimation from Satellite Images. PRRS 2021 - 11th IAPR International Workshop on Pattern Recognition in Remote Sensing, Jan 2021, Milan, Italy. pp.550-565, 10.1007/978-3-030-687878_40. hal-03066937

\section{HAL Id: hal-03066937 https://hal.science/hal-03066937}

Submitted on 15 Dec 2020

HAL is a multi-disciplinary open access archive for the deposit and dissemination of scientific research documents, whether they are published or not. The documents may come from teaching and research institutions in France or abroad, or from public or private research centers.
L'archive ouverte pluridisciplinaire HAL, est destinée au dépôt et à la diffusion de documents scientifiques de niveau recherche, publiés ou non, émanant des établissements d'enseignement et de recherche français ou étrangers, des laboratoires publics ou privés. 


\title{
Assessment of CNN-based Methods for Poverty Estimation from Satellite Images
}

\author{
Robin Jarry ${ }^{1,2[0000-0003-4118-3619]}$, Marc Chaumont ${ }^{2,3}$ [0000-0002-4095-4410], \\ Laure Berti-Équille ${ }^{4[0000-0002-8046-0570]}$, and Gérard Subsol ${ }^{2[0000-0002-7461-4932]}$ \\ 1 French Foundation for Biodiversity Research (FRB), Montpellier, France \\ 2 Research-Team ICAR, LIRMM, Univ. Montpellier, CNRS, Montpellier, France \\ 3 University of Nîmes, France \\ 4 ESPACE-DEV, Univ. Montpellier, IRD, UA, UG, UR, Montpellier, France
}

\begin{abstract}
One of the major issues in predicting poverty with satellite images is the lack of fine-grained and reliable poverty indicators. To address this problem, various methodologies were proposed recently. Most recent approaches use a proxy (e.g., nighttime light), as an additional information, to mitigate the problem of sparse data. They consist in building and training a CNN with a large set of images, which is then used as a feature extractor. Ultimately, pairs of extracted feature vectors and poverty labels are used to learn a regression model to predict the poverty indicators.

First, we propose a rigorous comparative study of such approaches based on a unified framework and a common set of images. We observed that the geographic displacement on the spatial coordinates of poverty observations degrades the prediction performances of all the methods. Therefore, we present a new methodology combining grid-cell selection and ensembling that improves the poverty prediction to handle coordinate displacement.
\end{abstract}

\section{Introduction}

Estimating poverty indicators is essential for the economy and political stability of a nation. These indicators are usually evaluated by surveying the population and asking people many questions. This requires a heavy organization, takes a long time, is sometimes only limited to easily accessible areas, and may be subjective. Therefore, solutions were proposed to estimate poverty at a large scale in an easier and faster way by using observational data, i.e., with no direct interaction with the people.

Satellite images give a quite precise observational overview of the planet. As early as 1980, Welch [Welch, 1980] monitored urban population and energy utilization patterns from the images of the first Landsat satellites. Since 1999 and 2013 respectively, Landsat 7 and 8 satellite series cover the entire Earth surface with a temporal resolution of 16 days and a spatial resolution of 15 to 30 meters depending on the spectral bands (8 for Landsat 7 and 11 for Landsat 8). Since 2014, Sentinel satellite series generate images with a spatial resolution of 10 meters. It is then possible to get remote-sensing data with a high time-frequency and a good spatial resolution.

Nevertheless, estimating the local wealth or poverty indicator rather than general value over a country is a very challenging problem. This requires the mapping of the 
observational data (i.e., the pixel intensity of a satellite image) and the poverty indicator precisely located. In some cases, numerous indicators can be available. For example, in [Babenko et al., 2017], 350,000 poverty indicators are collected in two surveys over the city of Mexico or, in [Engstrom et al., 2017], 1,300 ground-truth poverty indicators are obtained for a limited 3,500 $\mathrm{km}^{2}$ area of Sri Lanka. With a large number of indicators, direct supervised training with high-resolution daytime satellite images becomes possible, in particular with Deep-Learning techniques. Unfortunately, the number of such indicators is usually low in most countries, especially in developing ones. For example, only 770 poverty indicators are reported in 2016 for Malawi $^{5}$ with an area of more than 118,000 $\mathrm{km}^{2}$. In 2013, Chandy [Chandy, 2013] reported in general terms that almost two-fifths of the countries do not perform at least one income survey every 5 years. Based on this observation, a direct Machine-Learning approach, processing a set of geolocalized satellite images associated with the corresponding poverty indicators would inevitably lead to over-fitting, and then very bad generalization (e.g., see [Ngestrini, 2019]).

But, in 2016, Jean et al. [Jean et al., 2016] proposed an accurate and scalable method based on Deep-Learning to estimate local poverty from satellite imagery, based on sparse socio-economic data. They managed to solve the problem of the limited number of poverty indicators by using a Transfer Learning technique. It consists in training a Convolutional Neural Network (CNN) on one task and then applying the trained CNN to a different but related task. Since this seminal work, many papers have been published with either applying or extending the original algorithm, or propose some variants of Deep-Learning techniques. In particular, Ermon Group ${ }^{6}$ at Stanford University proposed different methods to tackle the problem (see [Yeh et al., 2020] for their latest results).

The difficulty is then to compare all the available methods as they do not use the same input data and they are not evaluated with the same set of parameters. In this paper, we propose to assess the methods with a common framework in order to analyze their performances, understand the limitations of the available data (both images and indicators), and evaluate whether combining them would improve the results. In Section 2, we describe three methods we selected in this line of research. In Section 3, we present the common framework to assess fairly the performances of the methods. In Section 4, we discuss adapting the methods to improve the performances. Conclusion and future work are presented in Section 5.

\section{Description of the Selected CNN-based Methods}

Transfer Learning emerged recently as an efficient way to deal with a limited number of poverty indicators. For our application, proxy data related to poverty and available in large quantities can be used to train a CNN for classifying satellite images accordingly.

By taking a specific layer of the resulting $\mathrm{CNN}$, we can extract a feature vector that characterizes the input satellite image with respect to its correlation with the corre-

\footnotetext{
${ }^{5}$ Living Standard Measurements Study, Malawi 2016: https://microdata.worldbank. org/index.php/catalog/2939.

${ }^{6}$ Ermon Group web page : https://cs.stanford.edu/ ermon/website/
} 
sponding proxy data. As proxy data are chosen to be related to poverty, we can assume that the feature vector is an appropriate representation of poverty in the image.

Finally, we can compute the feature vectors of satellite images at the sparse locations of the limited set of poverty indicators, and perform a regression between the vectors and the poverty indicators. This process can be done efficiently despite a limited number of locations and it can establish a relationship between the satellite images and the poverty indicators.

A major issue is then to select relevant proxy data. Several solutions have been proposed and we review three of them in the following sections.

\subsection{Nighttime Light}

The concept of using Transfer Learning to estimate poverty from satellite images was proposed in [Jean et al. 2016]. In this work, the authors used nighttime light values (NLV), which are known, since many years, to be a good proxy for poverty evaluation (see for example, [Elvidge et al., 1997], [Doll et al., 2000] or [Chen and Nordhaus, 2011]).

NLV data set is provided by the Earth Observation Group ${ }^{7}$ and the corresponding images are extracted from Google Static Map ${ }^{8}$, at 2.5 meters of resolution.

The authors introduce a CNN architecture to classify daytime satellite images by NLV. Three classes are used for low, medium, and high NLV [Jean et al., 2016, Supplementary Materials]. The CNN is based on a VGG-F architecture [Wozniak et al., 2018] and is pre-trained on the ImageNet dataset. Next, the network is fine-tuned on the training data set, which consists of 400,000 [Xie et al., 2016] images, each of them covering a $1 \mathrm{~km}^{2}$ area in Africa.

[Perez et al., 2017] is an extension of the previous research based on Landsat images. It shows that despite the lower resolution of Landsat images, the model accuracy is higher than the one of the previous benchmarks.

\subsection{Land Use}

In [Ayush et al., 2020], the authors assume that land use, and specifically the manufactured objects observed in a satellite image emphasize the wealthiness of an area. The authors trained a CNN on a land use detection and classification task, to extract a feature vector related to poverty. As proxy data, they used the $\mathrm{xView}$ data $\operatorname{set}^{9}$ consisting of very high-resolution images $(0.3 \mathrm{~m})$ annotated with bounding boxes defined over 10 main classes (building, fixed-wing aircraft, passenger vehicle, truck, railway vehicle, maritime vessel, engineering vehicle, helipad, vehicle lot, construction site) and 60 sub-classes. As the size of the objects in a satellite image may vary (e.g., a truck is much smaller than a building), the network used YoloV3, with DarkNet53 backbone architecture [Redmon and Farhadi, 2018], which allows the detection at 3 different scales. This network is pre-trained on ImageNet and fine-tuned on the $\mathrm{xView}$ data set. Since it was

\footnotetext{
${ }^{7}$ NOAA Earth Observation Group Website: https://ngdc.noaa.gov/eog/

${ }^{8}$ Google Static Map, getting started Web page: https://developers.google.com/maps/ documentation/maps-static/start.

${ }^{9}$ xView Challenge Website: http://xviewdataset.org/.
} 
trained on a detection/classification task, a single satellite image may have several detection results, depending on the number of objects in the image. Thus, the input image may be associated with multiple feature vectors, one for each detection. The authors further explore this additional information by different combinations of the feature vectors.

\subsection{Contrastive Spatial Analysis}

In [Jean et al., 2019], the idea is to be able to cluster homogeneous-looking areas and assume that some clusters will be specific to poor areas. Contrary to the two previous approaches, this method is based on an unsupervised task. A CNN model is trained with the aim of learning a semantic representation of an input image according to the following constraints: (i) If two sub-parts of an image are close spatially, they must have a similar semantic representation (i.e., feature vectors); and (ii) If two sub-parts of an image are far apart, they must have a dissimilar semantic representation.

The data set is a set of image triplets $(a, n, d)$, where $a, n$, and $d$ are tiles that are sampled randomly from the same image.

More precisely, the neighbor tile $n$ is sampled randomly in a given neighborhood of $a$, the anchor tile and $d$, the distant tile is sampled randomly outside of $a$ 's neighborhood. The objective is to minimize the following cost function using the CNN:

$$
L\left(t_{a}, t_{n}, t_{d}\right)=\max \left(0,\left\|t_{a}-t_{n}\right\|_{2}-\left\|t_{a}-t_{d}\right\|_{2}+m\right)
$$

where $t_{a}, t_{n}$ and $t_{d}$ are the feature vectors produced by the CNN for the anchor tile $a$, the neighbor tile $n$, and the distant tile $d$ respectively. $m$ is the margin to enforce a minimum distance. During the minimization process, if $t_{n}$ is too far from $t_{a}$ compared to $t_{d}$ (using the Euclidean distance), then $t_{n}$ will be generated to get closer to $t_{a}$, and $t_{d}$ will get more distant from $t_{a}$. The equilibrium is obtained when $t_{d}$ is in the hypersphere with radius $\left\|t_{a}-t_{n}\right\|+m$. The CNN is a modified version of ResNet-18 taking the three tiles as inputs.

\subsection{Regression Step}

All the previous methods aim to produce a feature vector representation of a satellite image that should represent poverty to a certain extend. The first approach uses a feature vector that estimates NLV from the satellite images. The second approach uses a feature vector that estimates land use. The third approach uses a feature vector that emphasizes the differences between two satellite images. Throughout the paper, we will name the three methods, Nighttime Light, Land Use, and Contrastive respectively. For each method, a Ridge regression model is trained with the pairs of feature vectors (obtained from the corresponding method) and the poverty indicators. The feature vectors are generated from the images of the sparse locations where some poverty indicators are available. We select the Ridge regression model, that is as close as possible to the methodology exposed in [Jean et al., 2016], [Jean et al., 2019], and [Ayush et al., 2020]. 


\subsection{Comparison Issues}

The three methods aforementioned may seem to offer comparable results from the results reported, but as a matter of fact, they differ greatly by the choice of the satellite images, proxy data, and feature vector parameters as we can see in Table 1.

\begin{tabular}{|l||ccc|}
\hline & Nighttime Light & Contrastive & Land Use \\
\hline Countries & $\begin{array}{c}\text { Uganda, Malawi, } \\
\text { Tanzania, Nigeria }\end{array}$ & Uganda & Uganda \\
\hline $\begin{array}{l}\text { Image } \\
\text { source }\end{array}$ & $\begin{array}{c}\text { Google Static Map } \\
\text { (DigitalGlobe) }\end{array}$ & Landsat-7 & DigitalGlobe \\
\hline Resolution & $2.5 \mathrm{~m}$ & $30 \mathrm{~m}$ & $0.3 \mathrm{~m}$ \\
\hline $\begin{array}{l}\text { Spectral } \\
\text { Bands }\end{array}$ & 3 (RGB) & $\begin{array}{c}5 \text { (RGB + 2 additional } \\
\text { spectral bands) }\end{array}$ & 3 (RGB) \\
\hline $\begin{array}{l}\text { Feature } \\
\text { vector size }\end{array}$ & 4,096 & 512 & $10-60$ \\
\hline
\end{tabular}

Table 1. Differences between the input data and parameters used in the three methods.

Notice that the images do not share the same resolution and the structures and objects captured in the images do not have the same scale. Moreover, in [Jean et al., 2019], the images contain 5 spectral bands, whereas the other methods process standard RGB images.

Experiments reported in [Perez et al., 2017] have shown that lower resolution images and additional spectral bands could influence poverty estimation. With this consideration in mind, we can assume that the image resolution and the number of spectral bands are significant features that must be commonly set before any method comparison.

Additionally, the size of the feature vectors generated by the CNN differs for each method. As it is difficult to know the influence of this parameter on the final $R^{2}$ score, we set the size to be identical across the different methods, to provide a fair comparison.

\section{A Common Framework to Assess the CNN-based Methods}

Our goal is to compare the three methods consistently and fairly with a common framework, to expose their benefits and limitations. Therefore, we decided to use the same satellite image source, the same image dimension and scale, the same regression steps, and the same socio-economic indicators. Doing so, we can compare which approach better emphasizes the poverty and if an unsupervised method, i.e., without proxy data, can outperform Nighttime Light or Land Use methods.

Before discussing the framework, it is important to notice that the poverty indicators given in the available surveys are artificially moved from their actual position, which may lead to biasing the results. 


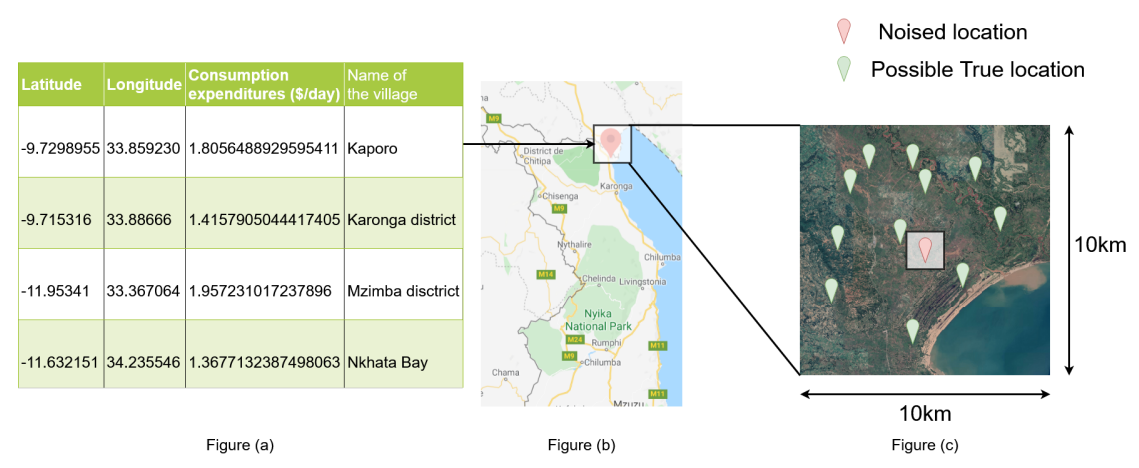

Fig. 1. Geographic displacement procedure performed on LSMS socio-economic surveys.

Surveying operators add a random geographic displacement up to $10 \mathrm{~km}$ on latitude and longitude from the location where the socio-economic indicator is evaluated, to protect the anonymity of respondents (see [Burgert et al., 2013 ${ }^{10}$ ). In the example given in Figure 1, the poverty indicators of a village are distributed over a $10 \mathrm{~km} \times 10 \mathrm{~km}$ surrounding area. This means that the actual poverty indicator can be significantly different from the reported one in the survey.

\subsection{Framework Specifications}

We can represent the three methods as the global pipeline shown in Figure 2. In the common framework we propose, the algorithm operates over a $10 \mathrm{~km} \times 10 \mathrm{~km}$ area around a given input location (i.e. latitude and longitude coordinates) with a corresponding poverty indicator. It takes as input a $4,000 \times 4,000$ pixels satellite image centered on the considered location. This ensures that the true indicator location is indeed in the image. Each image is then split into 100 sub-images (of $400 \times 400$ pixels), each one covering a $1 \mathrm{~km} \times 1 \mathrm{~km}$ area according to a regular grid. The sub-images are then processed by

10 This document shows how Demographic and Health Surveys are modified, and claims that the Living Standards Measurement Study, our survey provider, performs the same anonymization policy.

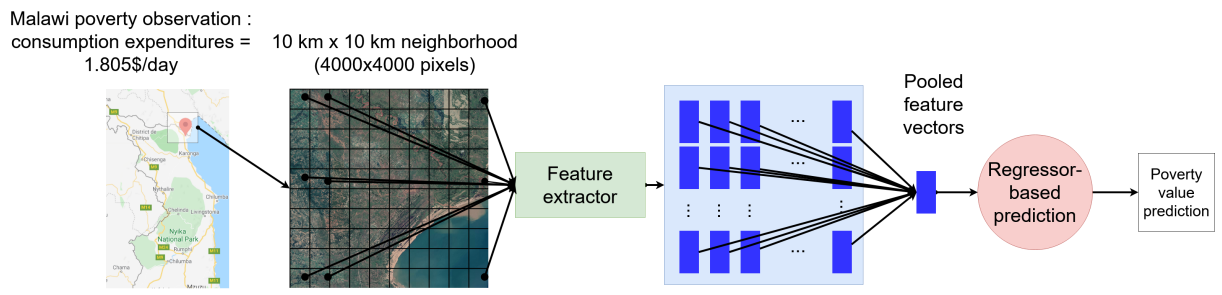

Fig. 2. Canonical pipeline for poverty prediction over $1 \mathrm{~km}^{2}$ areas. 
a Deep Learning feature extractor specific to a selected method (Nighttime Light, Land Use, Contrastive) in order to produce a fixed-size feature vector. Finally, the feature vectors are averaged and sent to a Ridge regressor, which returns the poverty estimation value for the $1 \mathrm{~km} \times 1 \mathrm{~km}$ area centered in the input location.

\subsection{Data Description}

Satellite images are used for both feature extractor training and poverty indicator estimation using regression. Some proxy data are also needed to build Nighttime Light and Land Use feature vectors.

Satellite images: Since we want to compare the approaches, the images used to build all the feature extractor and output the socio-economic estimates are provided by the Google Static Map API ${ }^{11}$. The satellite images used are $400 \times 400$ pixels, covering a $1 \mathrm{~km}^{2}$ area (2.5 meters resolution) with the classical RGB color channels. For each location in the survey, we use the same type of images to build a larger one that covers a $10 \mathrm{~km} \times 10 \mathrm{~km}$ neighboring area.

Proxy data for Nighttime Light method: As in [Jean et al., 2016], we used the $1 \mathrm{~km}$ resolution world map of NLV, provided by the Earth Observation Group in $2013^{12}$. Notice that afterward, the Earth Observation Group has provided 500m resolution world maps. Unfortunately, there is no reliable time reference mentioned and associated with Google Static Map images so we assume that 2013 NLV data are roughly consistent with Google Static Map images. Finally, 71,000 daytime images were randomly selected from the NLV world map in Africa.

Proxy data for Land Use feature extractor:

The $\mathrm{xView}$ dataset image resolution $(0.3 \mathrm{~m})$ is much higher than GSM one $(2.5$ $\mathrm{m}$ ), which prevents us from using the object detection methodology based on $\mathrm{xView}$ dataset. We then adapted the idea and use a global land use classifier which outputs a single value instead of a set of feature vectors corresponding to each detected object.

We used land use labels provided in the Eurosat data set [Helber et al., 2018]. It consists of almost 30,000 satellite images of European places, labeled into 10 classes: sea lake, river, residential, permanent crop, pasture, industrial, highway, herbaceous vegetation, forest, and annual crop. The authors provided a GeoTIFF data set ${ }^{13}$, including the locations of all images, we simply used these locations and downloaded the corresponding Google Static Map API images.

Proxy data for Contrastive feature extractor: Since it is an unsupervised method, no proxy data is required. Therefore, we used the poverty indicator location to generate $4,000 \times 4,000$, images with 2.5 meters resolution. Then, for each image, we randomly sample 100 anchor, neighbor, and distant image triplets, with the same size, area coverage, and channel as introduced earlier. We set the neighborhood size to 400 pixels, so

${ }^{11}$ Google Static Map getting started web page: https://developers.google.com/maps/ documentation/maps-static/start.

12 Nighttime Light world map: https://www.ngdc.noaa.gov/eog/dmsp/ downloadV4composites.html.

${ }^{13}$ GeoTIFF data set provided by [Helber et al., 2018]: https://github.com/phelber/ EuroSAT. 
the central pixel of each neighbor image (resp. distant image) is less (resp. more) than 400 pixels away from the anchor image. The overall process generates 77,000 triplets.

Poverty indicators: We used the poverty indicators from LSMS surveys ${ }^{14}$. We focus on the 2016 Malawi survey and consider the natural logarithm of the consumption expenditures as the poverty indicator. Note that the surveys are designed in two steps. The country is divided into clusters, and then, several households are surveyed within each cluster. In the resulting survey, only the clusters coordinates are reported (and randomly displaced for anonymization purposes, as explained earlier). In our experiments, we use the 770 clusters provided by the survey to train and test the poverty prediction models at the cluster level.

\subsection{Metrics and Evaluation Goal}

Performances of CNNs: We use the accuracy metric for assessing the classification task which allows us to compute feature vectors in Nighttime Light and Land Use. It is defined as follows:

$$
A=\frac{1}{N} \sum_{i=1}^{N} \delta\left(y_{i}, \hat{y}_{i}\right)
$$

with $y=\left(y_{i}\right)_{i \in\{1, \ldots, N\}}$ (resp. $\hat{y}=\left(\hat{y}_{i}\right)_{i \in\{1, \ldots, N\}}$ ) the true (resp. predicted) NLV value or land use, $N$ the number of predictions, and $\delta$ the Kronecker delta. Since Contrastive does not make any prediction, we can not use accuracy to measure its quality performance.

Performances of the Ridge regressor: We consider $y=\left(y_{i}\right)_{i \in\{1, \ldots, N\}}$ (resp. $\hat{y}=$ $\left(\hat{y}_{i}\right)_{i \in\{1, \ldots, N\}}$ ) as the true (resp. predicted) poverty indicator and $N$ be the number of predictions. Similarly to previous work, we used the $R^{2}$ score, also called coefficient of determination, defined as follows:

$$
R^{2}=1-\frac{\sum_{i=1}^{N}\left(y_{i}-\hat{y}_{i}\right)^{2}}{\sum_{i=1}^{N}\left(\bar{y}-y_{i}\right)^{2}} .
$$

$R^{2}$ aims to measure the mean squared error (numerator) over the variance (denominator). As a universal and classical metric, it corresponds to the amount of variance explained by the model.

Cross-validation: The usual train/test splitting process leads to too small data sets impacting the quality of the learning and decreasing the performances. The sparsity of poverty indicators causes some differences between training and testing data distributions. Therfore, the results obtained from a given train/test split can be very different from the ones obtained with other train/test splits. To avoid this problem, we use 10-fold cross-validation and average the scores.

\subsection{Implementation}

We ran our experiments using a workstation with $32 \mathrm{~Gb}$, Intel Xeon $3.6 \mathrm{GHz} 12$ cores, and a NVIDIA Quadro GV100 graphic card. We used the Python TensorFlow 2.x library.

\footnotetext{
${ }^{14}$ Living Standard Measurements Study, Malawi 2016: https://microdata.worldbank. org/index.php/catalog/2939.
} 
The three CNNs are based on the VGG16 architecture, followed by a fully connected layer (used to extract the features) and ended with the classification layer. The VGG16 architecture is pre-trained on ImageNet. We used stochastic gradient descent as optimizer.

All the image pixel values are scaled from 0 to 1 and a horizontal and vertical flip is randomly applied during the training phase as data-augmentation. At the end of each epoch, a validation step is done. The feature extractors are generated with the weights that optimize the validation accuracy when available (Nighttime Light and Land Use) or the validation loss (Contrastive). The training phase ran for 100 epochs and lasts for 18 hours for Nighttime Light, 13 hours for Land Use, and 3 hours for Contrastive.

The feature vectors generated by the feature extractors have $d=512$ dimensions. We use the Ridge regression with regularization parameters $\alpha$ chosen among the values $\left\{10^{-4}, 10^{-3}, \ldots, 10^{5}\right\}$.

\subsection{Results}

Table 2 gives $R^{2}$ scores which can be considered as baseline results. The first column contains the results obtained when only the feature vector corresponding to the anonymized position is used for regression (without considering the $10 \mathrm{~km} \times 10 \mathrm{~km}$ neighborhood). The second column contains the results obtained when all the feature vectors corresponding to an input image in the $10 \mathrm{~km} \times 10 \mathrm{~km}$ neighborhood are averaged. Regardless of the feature extractor, we can observe across the methods that there is a significant difference between the $R^{2}$ score when all the positions are considered compared to the score obtained with a single feature vector from the anonymized position. The gain ranges from +0.04 to +0.09 . Note that the $R^{2}$ values for the three state-of-the-art approaches are similar, with $0.47,0.46$, and 0.45 . Nevertheless, the $R^{2}$ standard deviation remains high and prevents a fair ranking of the approaches.

\begin{tabular}{|l|l|l|l|}
\cline { 2 - 4 } \multicolumn{1}{c|}{} & anonymized Position & All Positions & $\alpha$ \\
\hline Nighttime Light & $0.404 \pm 0.07$ & $0.449 \pm 0.09$ & $10^{2}$ \\
Land Use & $0.383 \pm 0.08$ & $\mathbf{0 . 4 7} \pm \mathbf{0 . 0 8}$ & $10^{3}$ \\
Contrastive & $0.385 \pm 0.07$ & $0.462 \pm 0.08$ & $10^{2}$ \\
\hline
\end{tabular}

Table 2. $R^{2}$ scores of the three poverty prediction methods, evaluated on the test data set (10-folds cross-validation).

\section{How to Improve the Results?}

Let us investigate two ways to ameliorate the methods' prediction. The first proposition consists in handling the spatial perturbation of the coordinates. The second approach consists in combining the three approaches. 


\subsection{Handling the Geographic Perturbation}

The random perturbation injected in the spatial coordinates of the surveyed poverty indicators may directly affect the prediction results. Therefore, we propose a 2-step method with two variants each, adapting the following step of the framework exposed in Section 3.1: For each position in the survey, (1) We consider all the $10 \mathrm{~km} \times 10 \mathrm{~km}$ neighboring areas for feature extraction, and (2) We average the resulting feature vectors.

On the one hand, the first step allows capturing the true position of the poverty indicator. On the other hand, it captures also the 99 false positions of the poverty indicator, perturbing the most important information. The second step reduces drastically the amount of information. The resulting average feature vector is a summarized representation of the $10 \mathrm{~km} \times 10 \mathrm{~km}$ neighborhood in which all the specific features are omitted.

Adaptation of Step (1) with grid-cell selection: Instead of considering all the $10 \mathrm{~km} \times 10 \mathrm{~km}$ neighboring areas for each position in the survey, we select specific cells in the grid of the neighborhood. The selection is based on the inhabitant number from WorldPop ${ }^{15}$ and NLV. More precisely, we keep the cells that correspond to: (a) The $n$ most populated places, and (b) The $n$ highest NLV, with $n$ varying in $\{1,5,10,20,40,60,80\}$.

After this first adaptation, we exclude "no mans' land" areas where there is no plausible position for a poverty indicator. Then, the feature extractors process only the selected cells and return $n$ feature vectors per position in the survey.

Adaptation of Step (2) without averaging the feature vectors: For each position in the survey: (a) We average the $n$ feature vectors and compare with the case where (b) The $n$ feature vectors are used for regression. In the latter case, the $n$ feature vectors are annotated with the poverty indicator collected at the corresponding position in the survey. Doing so, we ensure that the specific features captured in all the feature vectors are processed by the regression model.

\subsection{Combining the Approaches}

There is a large body of research on ensembling methods in the literature. As a starting point, we investigate two simple and straightforward ways of combining the approaches illustrated, in Figure 3. Future work will explore and test other ensembling methods.

Given the entire grid $(10 \mathrm{~km} \times 10 \mathrm{~km}$ area), a feature extractor outputs one vector for each cell. Please note that some cells may not be processed because of the gridcell selection, depending on their respective inhabitant number (1a) or NLV (1b). Given a grid-cell, i.e., a sub-image of $400 \times 400$ pixels that covers a $1 \mathrm{~km}^{2}$ area, each of the three feature extractors returns a feature vector. Then, the three feature vectors are either averaged (Ensembling Averaged) or concatenated (Ensembling Concatenated).

\subsection{Experiments and Analysis}

Performances of feature extractors: The CNNs are used to extract features from images. Therefore, their respective accuracy values are not used to determine whether or

15 WorldPop: https : //www . worldpop.org/geodata/summary?id=123 
not they are good classifiers. However, the accuracy metric can be used as an indirect measure to indicate the degree of consistency between the features generated by the CNNs, according to the classification problem (only for Nighttime Light and Land Use approaches). Nighttime Light reaches $64 \%$ of accuracy on the validation data set, whereas Land Use reaches $97 \%$. As mentioned earlier, instead of the accuracy measure, we use the $R^{2}$ score for evaluating Contrastive's performance on the poverty prediction task.

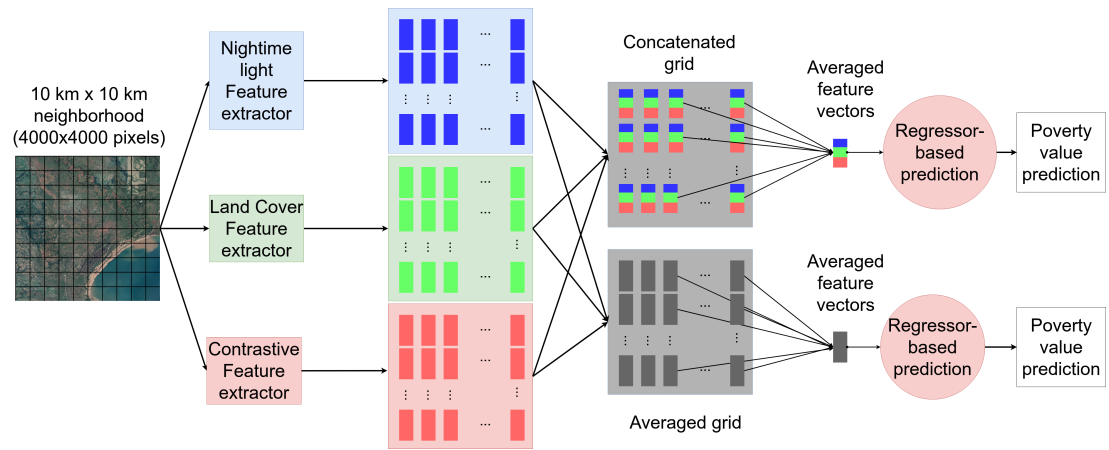

Fig. 3. Ensembling pipeline. Similar to figure 2, with an intermediate step of either concatenation or averaging, represented by the gray grids. Note that that the previous adaptation (1) and (2) are not shown, for the sake of clarity.

\begin{tabular}{|c|c|c|c|c|c|c|}
\hline & \multicolumn{2}{|c|}{$R^{2}$ of SOTA methods } & \multicolumn{4}{|c|}{$\begin{array}{c}R^{2} \text { of our methods handling geographic } \\
\text { perturbation }\end{array}$} \\
\hline & $\begin{array}{c}\text { Single } \\
\text { anonymized } \\
\text { position }\end{array}$ & $\begin{array}{l}\text { Averaging } \\
\text { across all } \\
\text { positions }\end{array}$ & $\begin{array}{l}\text { Population- } \\
\text { based } \\
\text { grid-cell } \\
\text { selection } \\
n=20 \text { and } \\
\text { averaging }\end{array}$ & $\begin{array}{l}\text { NLV-based } \\
\text { grid-cell } \\
\text { selection } \\
n=20 \text { and } \\
\text { averaging }\end{array}$ & $\begin{array}{c}\text { Random } \\
\text { grid-cell } \\
\text { selection } \\
n=20 \text { and } \\
\text { averaging }\end{array}$ & $\begin{array}{l}\text { Population- } \\
\text { based } \\
\text { grid-cell } \\
\text { selection } \\
n=20 \text { and } \\
\text { not } \\
\text { averaging }\end{array}$ \\
\hline $\begin{array}{l}\text { Nighttime } \\
\text { Light }\end{array}$ & $\mathbf{0 . 4 0 4} \pm \mathbf{0 . 0 7}$ & $0.449 \pm 0.09$ & $0.496 \pm 0.09$ & $0.487 \pm \mathbf{0 . 0 8}$ & $\mathbf{0 . 4 1 1} \pm \mathbf{0 . 0 7}$ & $\mathbf{0 . 3 7 9} \pm \mathbf{0 . 0 8}$ \\
\hline Land Use & $0.383 \pm 0.08$ & $0.47 \pm 0.08$ & $0.483 \pm 0.08$ & $0.466 \pm 0.08$ & $0.403 \pm 0.05$ & $0.35 \pm 0.07$ \\
\hline Contrastive & $0.385 \pm 0.07$ & $0.462 \pm 0.08$ & $0.486 \pm 0.04$ & $0.464 \pm 0.06$ & $0.388 \pm 0.05$ & $0.313 \pm 0.04$ \\
\hline $\begin{array}{l}\text { Ensembling } \\
\text { Concatenated }\end{array}$ & $0.44 \pm 0.08$ & $\mathbf{0 . 4 8} \pm \mathbf{0 . 0 9}$ & $0.491 \pm 0.07$ & $\mathbf{0 . 5 0 2} \pm \mathbf{0 . 0 8}$ & $0.429 \pm 0.07$ & $\mathbf{0 . 3 8 7} \pm \mathbf{0 . 0 7}$ \\
\hline $\begin{array}{l}\text { Ensembling } \\
\text { Averaged }\end{array}$ & $0.41 \pm 0.08$ & $0.47 \pm 0.09$ & $0.494 \pm 0.07$ & $0.487 \pm 0.07$ & $0.422 \pm 0.05$ & $0.378 \pm 0.07$ \\
\hline
\end{tabular}

Table 3. $R^{2}$ scores for different grid-cell selection (column) and feature extractors (row). Best $R^{2}=0.502$ is obtained with the ensembling method by concatenation, with NLV-based grid-cell selection $(n=20)$. 
Discussion on grid-cell selection: We can notice that the two grid-cell selection approaches have similar results, essentially because there is an overlap between the cells selected by each method.

When selecting the grid-cells, either based on the population criterion, or the NLV criterion, the average $R^{2}$ score increases up to +0.05 compared to the score obtained when using all the feature vectors. The highest increase is obtained with the population criterion. Each approach benefits from the population-based grid-cell selection. Only Nighttime Light benefits from NLV-based grid-cell selection. Compared to the results obtained by averaging all the feature vectors, all the approaches give an average $R^{2}$ above 0.48 . At this stage, we can say that our grid-cell selection method slightly improves the results. By selecting the most likely true positions of the indicators in the survey, we add the geographical context. However, when considering the same proportion of geographical context, random grid-cell selection is still less efficient than population-based grid-cell selection or NLV-based grid-cell selection, from +0.06 to +0.1 . This suggests naturally that capturing the true position improves the prediction of the model.

Discussion on averaging or not the feature vectors: With the same grid-cell selection, averaging the selected feature vectors leads to the best results and should be recommended. In Table 3, the same grid selection is applied for both columns 3 and 6 , but the selected feature vectors are averaged (column 3 ) and the selected feature vectors are all processed (column 6) by the regression model. We can notice that averaging increases the $R^{2}$ score up to +0.15 . The correct cell (correct position) with the correct poverty value may be masked by the 20 other feature vectors. This probably explains the poor performance of this approach.

Discussion on the ensembling approaches: Combining the approaches by concatenation or averaging always improves the average $R^{2}$ score by +0.01 approximately. The best results are obtained with NLV-based grid-cell selection, with an average $R^{2}$ reaching 0.50. However, the improvement is negligible. For example, when using the population-based grid-cell selection, there is no improvement compared to Nighttime Light alone.

Next, we investigate whether an ensembling approach combining the three methods can significantly improve the results. For several test folds and all the ground truth data in each test fold, we compare the relative prediction error made by Nighttime Light, Land Cover, and Contrastive (shown in Figure 4, for one particular test fold). Then, for each ground truth observations, we select the prediction with the smallest prediction error. We average the $R^{2}$ score obtained with the best prediction over the test folds, and obtain $R^{2}=0.6$. This score is significantly higher than all the other results presented in Table 3 . Thus, there is room for improvement by ensembling the methods.

Additionally, in Figure 4, we observe that, regardless of the feature extractor, the three approaches are slightly over-estimating the richness when the poverty is extreme, and give more erroneous predictions when the poverty is above $2.4 \$$ day. This can be explained by a fewer number of poverty indicators that are greater than $2.4 \$ /$ day in the learning set. 


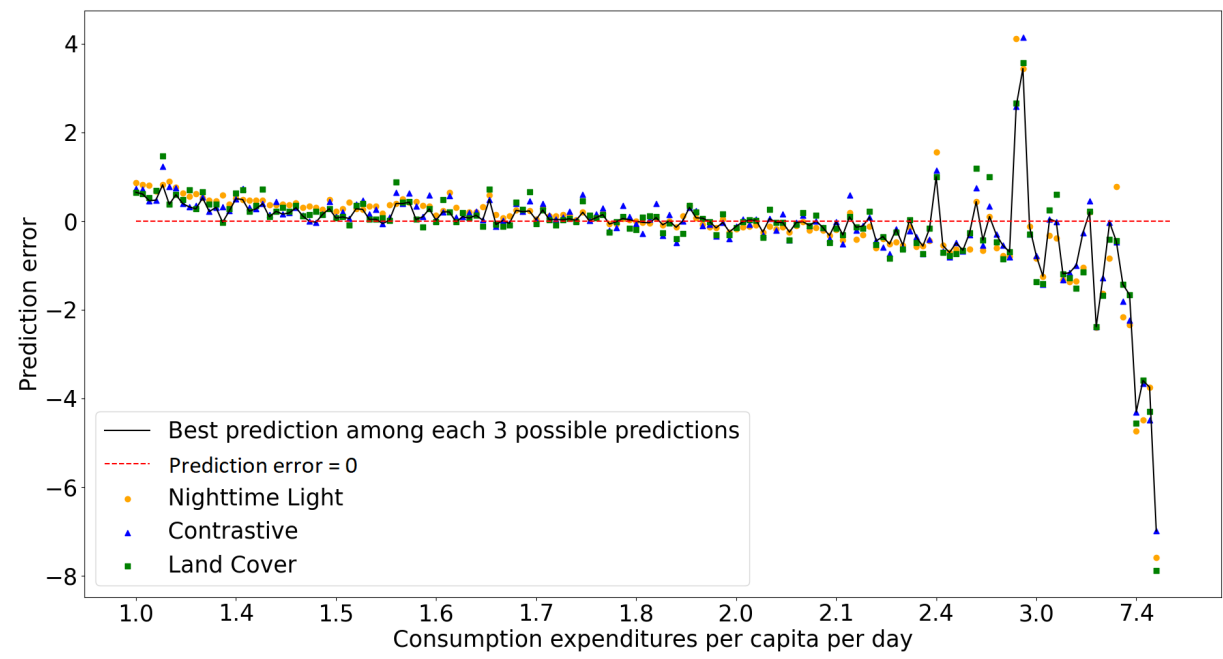

Fig. 4. Prediction error of the three feature extractors for one of the test fold with respect to the poverty level. The prediction is obtained by averaging all the feature vectors for a given position and applying the learnt regression model. Black line connects the best predictions among the three predictions for each test sample. Note that the $\mathrm{X}$-axis is not linear.

\section{Conclusion and Future Work}

By providing a fair comparison between three state-of-the-art models for poverty prediction, we present several insightful results: (1) Such models produce similar results on the same framework. (2) The spatial perturbation injected in the coordinates of poverty indicators is a key issue that reduces significantly the prediction power of the models. By handling the spatial perturbation of the coordinates, our grid-cell selection method shows better performances and motivates future work to ameliorate the entire process. (3) Using anonymized coordinates from the surveys implies poverty prediction at a $10 \mathrm{~km} \times 10 \mathrm{~km}$ scale (which can still capture the true position) rather than $1 \mathrm{~km} \times$ $1 \mathrm{~km}$ scale. However, it prevents the use of very high-resolution images available today. Covering a $100 \mathrm{~km}^{2}$ area with high-resolution images would necessitate very large images $(4,000 \times 4,000$ with 2.5 meters resolution) that cannot be directly processed by a CNN. We believe that this opens several new research directions to handle noise and high-resolution images for CNN-based prediction models. (4) We showed that our combination of the three considered methods does not augment drastically the quality performance. However, we experience that choosing the best prediction among the three methods leads to $R^{2}=0.6$. Thus, we claim that there exists a combination of the three methods that can give significant improvement of the $R^{2}$ score. As it is a choice on the prediction strategy, we believe that other or more sophisticated ensembling methods can be even more efficient. (5) Finally, the small size of the data set causes some differences and discrepancies between the training and testing data sets, which are reflected by high standard deviation values during the cross-validation. 
To pursue this work, we aim to propose a combination method that can reach higher $R^{2}$ scores, eventually using multi-spectral imagery to estimate poverty indicators as other work in the literature. Using the fair benchmark for poverty prediction we proposed, we plan to explore the benefits of multi-spectral images compared to natural RGB images. Finally, other deep learning architectures may be more likely to give a better feature representation of images. Therefore, one of our goals will be to test and find an optimal architecture.

\section{Acknowledgment}

This research was partly funded by the PARSEC group, funded by the Belmont Forum as part of its Collaborative Research Action (CRA) on Science-Driven e-Infrastructures Innovation (SEI) and the synthesis centre CESAB of the French Foundation for Research on Biodiversity. This work was also partly funded by the French ANR project MPA-POVERTY.

\section{References}

[Ayush et al., 2020] Ayush, K., Uzkent, B., Burke, M., Lobell, D., and Ermon, S. (2020). Generating interpretable poverty maps using object detection in satellite images. In Bessiere, C., editor, Proceedings of the Twenty-Ninth International Joint Conference on Artificial Intelligence, IJCAI-20, pages 4410-4416. International Joint Conferences on Artificial Intelligence Organization. Special track on AI for CompSust and Human well-being.

[Babenko et al., 2017] Babenko, B., Hersh, J., Newhouse, D., Ramakrishnan, A., and Swartz, T. (2017). Poverty mapping using convolutional neural networks trained on high and medium resolution satellite images, with an application in mexico. NIPS 2017 Workshop on Machine Learning for the Developing World.

[Burgert et al., 2013] Burgert, C. R., Colston, J., Roy, T., and Zachary, B. (2013). Geographic displacement procedure and georeferenced data release policy for the demographic and health surveys. DHS Spatial Analysis Reports No. 7. Calverton, Maryland, USA: ICF International.

[Chandy, 2013] Chandy, L. (2013). Counting the poor: Methods, problems and solutions behind the $\$ 1.25$ a day global poverty estimates. Working Paper No. 337682, Developement Initiatives \& Brooking Institution. http://devinit.org/wp-content/uploads/2013/09/ Counting-the-poor11.pdf.

[Chen and Nordhaus, 2011] Chen, X. and Nordhaus, W. D. (2011). Using luminosity data as a proxy for economic statistics. Proceedings of the National Academy of Sciences, 108(21):85898594.

[Doll et al., 2000] Doll, C. N. H., Muller, J.-P., and Elvidge, C. D. (2000). Night-time imagery as a tool for global mapping of socioeconomic parameters and greenhouse gas emissions. Ambio, 29(3):157-162.

[Elvidge et al., 1997] Elvidge, C., Baugh, K., Kihn, E., Kroehl, H. W., Davis, E., and Davis, C. (1997). Relation between satellite observed visible-near infrared emissions, population, economic activity and electric power consumption. International Journal of Remote Sensing, 18:1373-1379.

[Engstrom et al., 2017] Engstrom, R., Hersh, J., and Newhouse, D. (2017). Poverty from space: Using high-resolution satellite imagery for estimating economic well-being. Policy Research Working Paper; No. 8284, World Bank, Washington, DC. https : //openknowledge. worldbank .org/handle/10986/29075 License: CC BY 3.0 IGO. 
[Helber et al., 2018] Helber, P., Bischke, B., Dengel, A., and Borth, D. (2018). Introducing eurosat: A novel dataset and deep learning benchmark for land use and land cover classification. In IGARSS 2018-2018 IEEE International Geoscience and Remote Sensing Symposium, pages 204-207. IEEE.

[Jean et al., 2016] Jean, N., Burke, M., Xie, M., Davis, W. M., Lobell, D. B., and Ermon, S. (2016). Combining satellite imagery and machine learning to predict poverty. Science, 353(6301):790-794.

[Jean et al., 2019] Jean, N., Wang, S., Samar, A., Azzari, G., Lobell, D., and Ermon, S. (2019). Tile2vec: Unsupervised representation learning for spatially distributed data. In AAAI-19/IAAI19/EAAI-20 Proceedings, pages 3967-3974. AAAI technical track : Machine Learning.

[Ngestrini, 2019] Ngestrini, R. (2019). Predicting poverty of a region from satellite imagery using cnns. Master's thesis, Departement of Information and Computing Science, Utrecht University. Unpublished, http://dspace.library .uu.nl/handle/1874/376648.

[Perez et al., 2017] Perez, A., Yeh, C., Azzari, G., Burke, M., Lobell, D., and Ermon, S. (2017). Poverty prediction with public landsat 7 satellite imagery and machine learning. NIPS 2017, Workshop on Machine Learning for the Developing World.

[Redmon and Farhadi, 2018] Redmon, J. and Farhadi, A. (2018). Yolov3: An incremental improvement. CoRR, abs/1804.02767.

[Welch, 1980] Welch, R. (1980). Monitoring urban population and energy utilization patterns from satellite data. Remote Sensing of Environment, 9(1):1 - 9.

[Wozniak et al., 2018] Wozniak, P., Afrisal, H., Esparza, R., and Kwolek, B. (2018). Scene recognition for indoor localization of mobile robots using deep CNN. International Conference on Computer Vision and Graphics.

[Xie et al., 2016] Xie, M., Jean, N., Burke, M., Lobell, D., and Ermon, S. (2016). Transfer learning from deep features for remote sensing and poverty mapping. 30th AAAI Conference on Artificial Intelligence.

[Yeh et al., 2020] Yeh, C., Perez, A., Driscoll, A., Azzari, G., Tang, Z., Lobell, D., Ermon, S., and Burke, M. (2020). Using publicly available satellite imagery and deep learning to understand economic well-being in Africa. Nature Communications, 11(1):2583. 